\title{
Study of the Effects of Alcohol on Drivers and Driving Performance on Straight Road
}

\author{
Xiaohua Zhao, Xingjian Zhang, and Jian Rong \\ Key Lab of Traffic Engineering, Beijing University of Technology, Beijing 100124, China \\ Correspondence should be addressed to Xiaohua Zhao; zhaoxiaohua@bjut.edu.cn
}

Received 26 November 2013; Revised 14 January 2014; Accepted 16 January 2014; Published 23 February 2014

Academic Editor: Wuhong Wang

Copyright ( 2014 Xiaohua Zhao et al. This is an open access article distributed under the Creative Commons Attribution License, which permits unrestricted use, distribution, and reproduction in any medium, provided the original work is properly cited.

\begin{abstract}
Drinking driving is responsible for a high proportion of traffic accidents. To study the effects of alcohol on drivers and driving performance, 25 drivers' subjective feelings and driving performance data in different blood-alcohol concentration (BAC) levels were collected with simulated driving experiment. The investigation results revealed that alcohol affected drivers in many aspects, including attitude, judgment, vigilance, perception, reaction, and controlling. The analysis of accident rate showed that higher BAC level would lead to higher accident rate. The statistical analysis results of driving performance indicated that average speed, speed standard deviation, and lane position standard deviation were significantly higher under the influence of alcohol. They also had a statistically significant linear trend as the function of BAC level. The discrimination of drinking driving based on driving performance was performed with Fisher discrimination method. The results showed that drinking driving with higher BAC level was easier to discriminate from normal driving. Also, the results indicated that the three significant indicators on straight roadway could be used in the discrimination of drinking driving state. The conclusions can provide references for the study of drinking driving and the identification of driving state and then contribute to traffic safety.
\end{abstract}

\section{Introduction}

It has been known that alcohol use impairs driving skills and increases accident risk. It has been found that while driving under the influence of alcohol, the risk of having an accident causing injury or death increases exponentially [1]. In Europe, drinking driving is thought to be responsible for 10,000 deaths each year [2]. Alcohol-impaired driving accidents contribute to approximately $31 \%$ of all traffic fatalities in the USA [3]. In China, Li et al. revealed that about $34.1 \%$ of road accidents were alcohol related [4].

Drunk driving has a high probability to lead to serious accidents. Even with a small amount of alcohol assumption, drivers are twice likely to be involved in traffic accidents than sober drivers [5]. Therefore, many countries have been working on solutions to drunk driving for a long period of time, including publicity and education and tough drunkdriving laws. The laws have been enacted to prohibit driving after drinking and have imposed severe penalties on violators [6]. The legal limits for BAC are between $0.01 \%$ and $0.08 \%$.
The limit, for example, is $0.02 \%$ in Sweden; $0.05 \%$ in Israel, Korea, and Australia; and 0.08\% in Canada, England, Mexico, and the United States. In China, driving with a BAC higher than $0.02 \%$ is defined as drink-driving and the driver will be penalized. Moreover, driving with a BAC higher than $0.08 \%$ is considered drunken-driving and it belongs to unlawful act.

Even so, drinking driving is still difficult to be completely eliminated. It is also needed to analyze the signatures of alcohol's impairment on drivers to pave the foundation for the study on the countermeasure of drinking driving. Alcohol can affect drivers' cognition, vigilance, attention, judgment, and reaction, which were related to driving ability closely. It was concluded that alcohol consumption, even at low doses, significantly affected driving-related skills such as vision, braking behavior, and vigilance [7]. At the same time, drivers' information process and attention were heavily affected by alcohol. Nash demonstrated that drivers would be distracted by alcohol when they were asked to complete some tasks [8]. It has been indicated that alcohol would negatively affect drivers' ability on judgment of following distance and depth 
perception [9]. Alcohol's effects on visual performance are most obvious when it comes to the judgment of moving objects and the process of different information at the same time $[10,11]$. Williamson et al. [12] found that alcohol affected many parameters for a long time, including simple reaction times, vigilance, visual searches, and logical reasoning.

Alcohol not only affects the parameters mentioned above but also causes decline in driving performance. Linnoila et al. [13] indicated that a driver's ability to operate a vehicle was affected at a BAC level of $0.035 \%$. Alcohol also decreases hand steadiness [14] and operating accuracy at a BAC level of $0.06 \%$ [15]. Some researchers have found that alcohol impairs behaviors such as steering and braking at BACs ranging from $0.05 \%$ to $0.10 \%[16,17]$. It has been proved that alcohol could impair steering and braking control ability [9]. Fillmore et al. [18] expressed that alcohol significantly impaired driving performance, which included deviation of lane position, line crossings, steering rate, and driving speed. Chamberlain and Solomon [7] concluded that alcohol consumption negatively affects steering wheel control and braking behavior. In other words, there is unequivocal evidence that alcohol significantly impairs driving performance, as demonstrated through laboratory, simulator, and driving studies.

Although there have been some researches about the alcohol impairment, few studies explained the detailed characteristics of the impairment and driving performance at different BAC levels. At the same time, fewer researches discriminated drinking driving based on driving performance. Discrimination with driving performance, which is the noncontact method, may have a greater potential for application. To summarize the effect of alcohol on drivers and driving performance, drivers were recruited to conduct simulation driving experiment at different BAC levels. In this paper, we mainly paid attention to the driving performance on urban straight roadway segment, which was the most common road in driving process. From the experiment, participants' subjective feelings were investigated to summarize the effect on driving state, and the parameters of vehicle's travelling condition were collected to analyze the signatures of driving performance. Based on the significant indicators of driving performance, drinking driving state was tried to identify normal state. The objectives of this paper are mainly the following: (1) to summarize signatures of drivers' subjective feelings under the influence of alcohol, (2) reveal the effects of drinking driving at different BAC levels on driving performance, and (3) try to identify driving state based on the indicators of driving performance. The outcomes are expected to provide references for discrimination of drinking driving state and to guide the study of accident prevention caused by drinking driving.

\section{Materials and Method}

2.1. Participant. Nagoshi et al. [19] indicated that male drivers, affected by drinking driving, were more impulsive and sensation-seeking than female. It has also been found that for the same level of BAC, young drivers have a higher relative accident risk than older drivers $[20,21]$. Thus, a total of 25

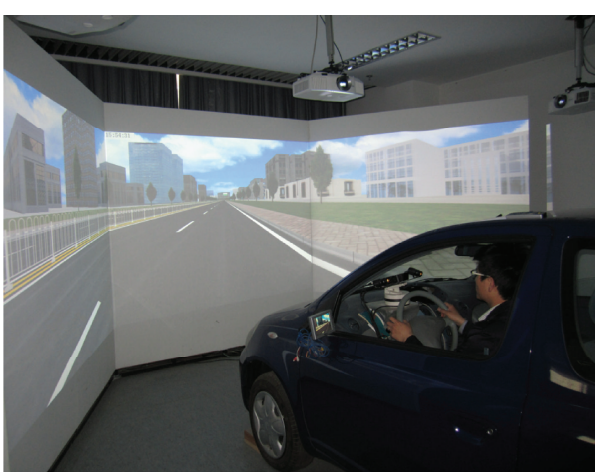

FIGURE 1: Driving simulator.

healthy young male drivers were recruited to participate in this research. The average age of them was $25(\mathrm{SD}=4.1$, range $=20-35$ years). All the participants possess valid driver's licenses more than 3 years (average $=3.6$ ). All the participants had regular driving habits, stationary sleep time, and no drug use. They agreed and signed an informed consent before participating in the study and were paid for the experiment.

2.2. Equipment. Because of the risks of drinking driving, the experiments were performed based on a driving simulator. The validity of using the driving simulator for the study has been researched in depth by Bella [22, 23]. Driving simulations were performed with the AutoSim driving simulator system. The simulator consists of six network computers and some operation hardware interfaces, including steering wheel, three pedals, and manual gearshift. The road scenario is projected onto three big screens in front providing a 130 degree field of view, with two rearview mirrors on each side and one screen on the back. The width of the vehicle was about 1.8 meters. The simulator can record the degree of the actions of the driver stepping on three pedals (brake, throttle, and clutch), the steering wheel angle, and the gear state. Additionally, the simulator provides many other parameters that describe vehicle's traveling conditions, including the traveling speed, lane position, displacement, and acceleration. The sampling frequency of driving simulator in this experiment is $30 \mathrm{~Hz}$. The driving simulator was shown in Figure 1.

In the experiment, a blowing-type alcohol detector was used to test drivers' BAC level. The detector is the same type as the one used by traffic police in Beijing. The BAC level of participants was measured five times each testing and the mean BAC level was used to minimize measurement errors.

2.3. Scenario. The scenario was designed as four-lane bidirectional urban road, including 5 urban straight roadway segments and 6 urban curves. The width of the road lane was 3.75 meters. The straight roadway and curve were alternant in the scenario. Each urban straight roadway segment was 1000 meters long. Considering that driver would accelerate or decelerate, we considered the middle 800 meters an urban straight roadway segment, which was the object of study. Three similar scenarios were designed for the study to avoid participants' familiarity with only one route, and a random 


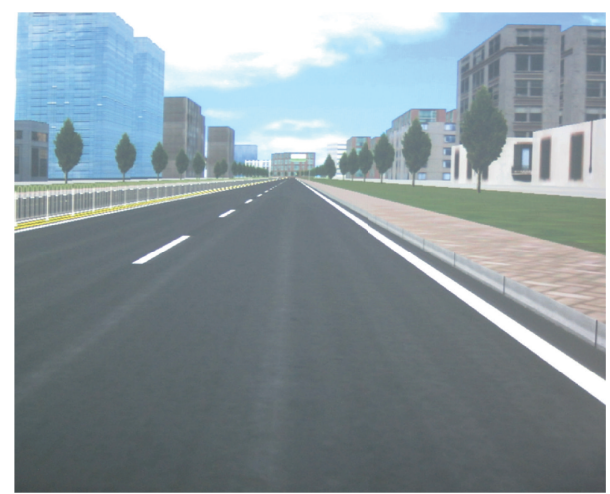

Figure 2: Scenario.

scenario was selected for each simulated driving. To avoid the interference of other vehicles, the scenario was designed without any other vehicles. Then participants can drive freely in the road. The designed scenario was shown in Figure 2.

2.4. Procedure. To analyze the effect of alcohol on driving performance at different level, participants were required to conduct experiments at three different BAC levels. The low BAC level was set around $0.03 \%$, which was classified as drink-driving according to Chinese law. The high BAC level was set higher than $0.09 \%$, which was classified as drunkdriving. The BAC level of $0.06 \%$ was set in the middle between low and high BAC level. Another driving state was the normal state, which was considered the control state. According to the driving state design, each participant performed the simulated driving experiment four times in four different days. To avoid residual effects of alcohol dose, the participants carried out the experiments at four BAC levels of $0.00 \%$, $0.03 \%, 0.06 \%$, and $0.09 \%$ at intervals of 3,5 , and 7 days, respectively.

During the experiment, the alcohol dose of each participant was calculated according to Watson's research [24]. Equation (1) can be used to calculate the dose for the expected BAC level:

$$
\begin{aligned}
\text { Alcohol dose }(\mathrm{g})= & {\left[\frac{10 \times \mathrm{BAL} \times \mathrm{TBW}}{0.8}\right]+10 } \\
& \times \mathrm{MR} \times(\mathrm{DDP}+\mathrm{TPB}) \times\left(\frac{\mathrm{TBW}}{0.8}\right),
\end{aligned}
$$

where BAL is the target blood-alcohol level, TBW is the total body water amount, MR is the metabolic rate (generally $0.015 \mathrm{~g} / 100 \mathrm{~mL} / \mathrm{h}), \mathrm{DDP}$ is the duration of the drinking period, and TPB is the time to peak BAL (generally $0.5 \mathrm{~h}$ ). Generally, TBW for men is as follows:

$$
\begin{aligned}
\text { Men's TBW }= & 2.447-0.09516 \times \text { Age }+0.1074 \\
& \times \text { Height }(\mathrm{cm})+0.3362 \times \text { Weight }(\mathrm{kg}) .
\end{aligned}
$$

Chinese liquor (46\% of alcohol content) was used for the drinking. With each drink, the dose was first calculated according to the equations above for each subject. Then the liquor was mixed into water to make that integral dose be $500 \mathrm{~mL}$ and the participant did not know how much alcohol he drank. About 15 minutes after drinking, participants' BAC level was measured every 5 minutes. When they achieved the target BAC levels, the simulated driving began. The procedure of the four times experiments was shown in Table 1.

Each participant was asked to perform the four visits with a random order to balance the effect of the order on drivers. All the participants were required to sleep well at least three days before experiments and refrain from having any stimulating food or beverage. They were also asked to have a noon break at least one hour on the day of the experiment. At the beginning of the experiment, participants had to meet the requirements of normal state to get experiment data without interference of other states. In the first time experiment, subjects were instructed regarding the operation of the simulator, the experimental procedure, and the tasks to be performed. Then, they were given approximately 10 minutes to practice driving to familiarize themselves with the simulator control and the road environment.

The experiments were carried out after 2:00 pm each time, when the participants were not sleepy according to a conventional sleep cycle. In each time, subjects were invested about their state before simulated driving experiment to ensure that they were not affected by any other unexpected factors. They were asked to have a break about 5-10 minutes in middle of simulated driving process to avoid fatigue. Each subject drove in two random scenarios and therefore had 10 urban straight roadway segments data. After simulated driving, participants were required to fill in the questionnaire, which included attitude, vigilance, attention, judgment, reaction, and ability of controlling vehicle.

2.5. Data Collection and Analysis. Based on the driving simulator, 22 of the 25 participants' data of speed and lane position of the vehicle's travelling condition controlled by them at different BAC levels were collected effectually. The two parameters were considered the representative of driving performance. Lane position was defined as the distance between the center of the vehicle and the lane line on the right. Considering that the width of road lane was $3.75 \mathrm{~m}$, the best lane position was $1.875 \mathrm{~m}$. At the same time, all the subjects completed successfully the questionnaire and the data were collected integrally. The investigation included 9 aspects of drivers' subjective feelings: safety attitude, driving attitude, vigilance, attention, speed-sense, direction-sense, judgment ability, ability of controlling direction, and reaction capacity. Participants were investigated after drinking driving with BAC level of $0.09 \%$. They were required to fill out the questionnaire contrasting with their feelings when normal driving. Each aspect of the investigation included three options, which meant worse, invariant, and better, respectively, contrast with normal driving. For example, the three options for safety attitude were adventurous, invariant, and cautious. 
Table 1: Procedure of experiment.

\begin{tabular}{lllll}
\hline & Visit 1 & Visit 2 & Visit 3 & Visit 4 \\
\hline Goal state & Normal & Low BAC level & Middle BAC level & High BAC level \\
Time & $2: 00 \mathrm{pm}$ & $2: 00 \mathrm{pm}$ & $2: 00 \mathrm{pm}$ & $2: 00 \mathrm{ppm}$ \\
Step 1 & Test drive & Drink to BAC level $=$ & Drink to BAC level = & Drink to BAC level = \\
& & $0.03 \%$ & $0.06 \%$ & $0.09 \%$ \\
Step 2 & Questionnaire survey & Questionnaire survey & Questionnaire survey & Questionnaire survey \\
& about state & about feeling & about feeling & about feeling \\
Step 3 & Simulated driving & Simulated driving & Simulated driving & Simulated driving \\
& about 40 min & about 40 min & about 40 min & about 40 min \\
Step 4 & Questionnaire survey & Questionnaire survey & Questionnaire survey & Questionnaire survey \\
& about driving process & about driving feeling & about driving feeling & about driving feeling \\
\hline
\end{tabular}

TABLE 2: Accident rate at different BAC levels.

\begin{tabular}{lcccc}
\hline State & Normal & BAC $=0.03 \%$ & BAC $=0.06 \%$ & BAC $=0.09 \%$ \\
\hline Accident rate & $1.51 \%$ & $5.22 \%$ & $6.96 \%$ & $8.70 \%$ \\
\hline
\end{tabular}

The objective of the analysis was to explore the signatures of alcohol impairment. The data was analyzed according to the following method.

(i) First, the signatures of drivers' subjective feelings under the influence of alcohol were summarized with distribution proportion analysis.

(ii) Second, we count up the number of accidents and the accident rate to illustrate dangers at different BAC levels.

(iii) Third, the data of the accident-occurred sections was removed due to the vehicle's stopping. The indicator value of each participant was the mean of all his driving sections excluding accident sections. ANOVA with repeated measures was used to analyze the differences of each indicator at the four states. The pairwise comparison was analyzed using post hoc $t$ test.

(iv) At the end, Fisher discrimination method with the indicators of driving performance was used to identify the state of drinking driving from normal driving. Firstly, all the drinking driving states at different BAC levels were put in one group to identify normal driving state without considering different BAC levels; then, drinking driving states with different BAC levels were separately distinguished from normal driving state.

\section{Results}

3.1. Signatures of Drivers' Subjective Feelings. Nine aspects of drivers' subjective feelings were investigated with questionnaires in the experiment. Compared with normal state, drivers described their feelings under the influence of alcohol after drinking simulation driving. The distribution of the investigation results for 25 subjects was shown in Figure 3. The distribution about safety attitude, for example, meant that $60 \%$ of the 25 participants (15) were more adventurous when drinking driving than normal driving and $24 \%$ of them (6) were more cautious.

The driving attitude and safety attitude showed driving tendencies of drivers. It indicated that most subjects admitted that they had the tendency of sensation-seeking and were more adventurous under the influence of alcohol. It was revealed in Figure 3 that all of the abilities on perception, attention, direction-sense, judgment, controlling, and reaction of most drivers were impaired by alcohol. More than half of subjects felt that the vehicle moved slowly, which might make them drive much faster.

3.2. Accident Rate Analysis. Each participant drove 10 straight roadway segments and each section was 800 meters long. There were some accidents in their simulated driving process. Some drivers rush out or drove into the guardrail in center of the road. At different driving states, we defined accident rate that the ratio of the total number of the segments including accidents of all participants divided the total number of urban straight roadway segments of all participants had driven. The accident rate at different states was shown in Table 2. The accident rate $1.51 \%$, for example, meant that there were about 200 sections in total in the experiment and accidents occurred in about 3 sections. Table 2 showed that the higher BAC level induced higher accident rate, which indicated that driving ability was impaired more seriously at higher BAC level.

3.3. Signature of Driving Performance. Driving performance data was analyzed to reveal the effect characteristics of drunk driving on running vehicle. Four indicators of vehicle travelling conditions, average speed (SP_AVG), speed standard deviation (SP_SD), average lane position (LP_AVG), and lane position standard deviation (LP_SD), were used to explain the signatures of driving performance impaired by alcohol. The means of all subjects of each indicator at different driving states were shown in Figure 4. 


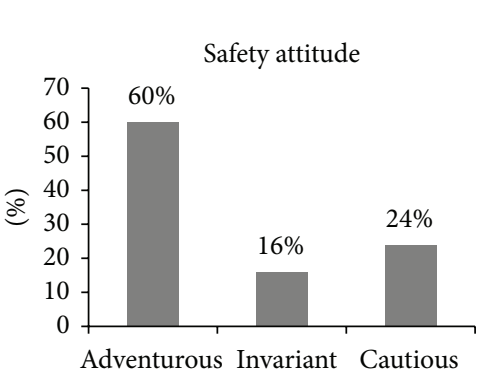

(a)

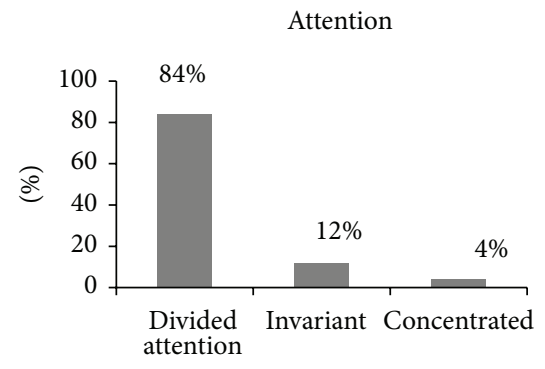

(d)

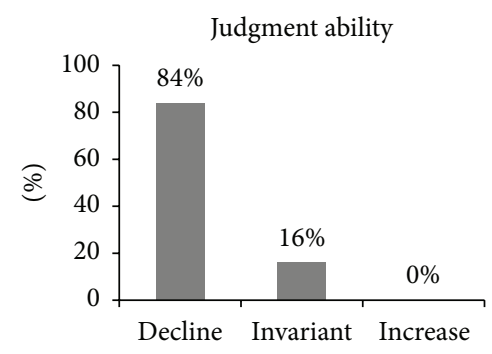

(g)

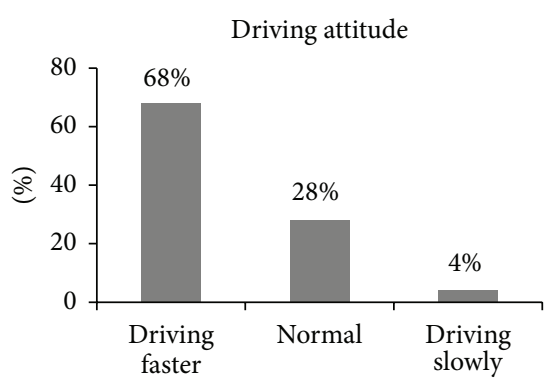

(b)

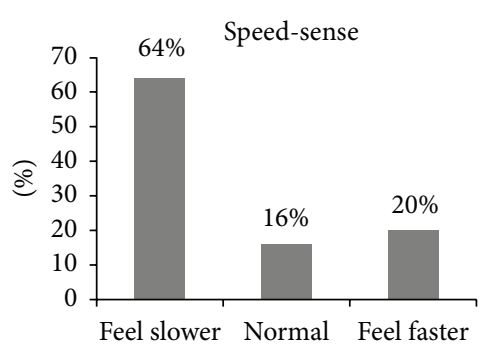

(e)

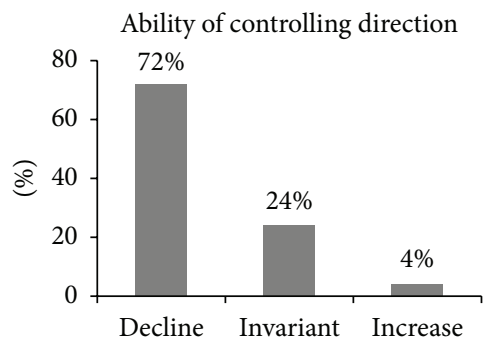

(h)

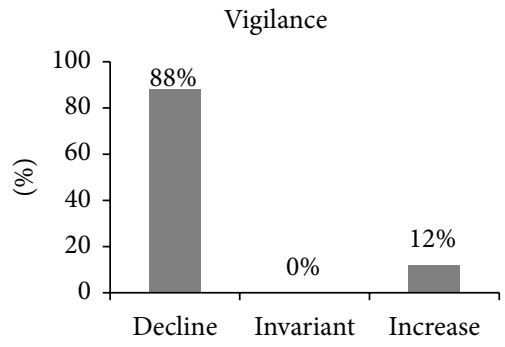

(c)

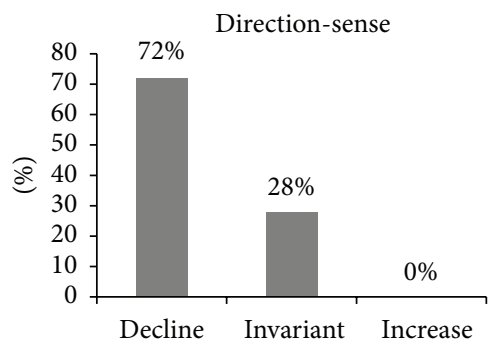

(f)

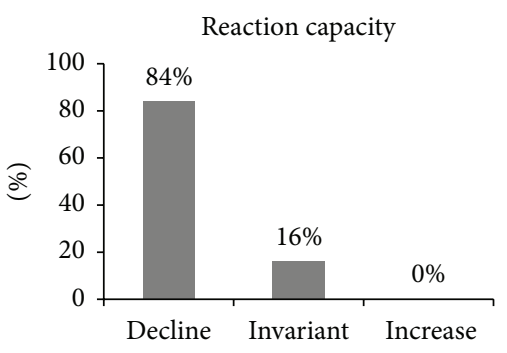

(i)

FIGURE 3: Distribution proportion of drivers' subjective feelings under the influence of alcohol compared with normal state.

The main differences between normal driving and drinking driving were analyzed with ANOVA method firstly. The results showed that SP_AVG was significantly higher in drinking driving state than in normal state $(F(1,86)=4.321$, $P<0.05)$. So do SP_SD $(F(1,86)=5.199, P<0.05)$ and LP_SD $(F(1,86)=4.386, P<0.05)$. No significant difference was found about LP_AVG.

Then, ANOVA with repeated measure was used to analyze the four indicators at different BAC levels. Mauchly's test of sphericity for SP_AVG was statistically significant, and the Greenhouse-Geisser adjustment was used to adjust the degree of freedom. Of the within-subjects effects, the main effect of BAC was statistically significant, $F(2.263,47.519)=$ 4.461, $P<0.05$, and partial $\eta^{2}=0.175$. As shown in Figure 4(a), SP_AVG increased as a function of BAC, producing a statistically significant linear trend, $F(1,21)=$ 8.929, $P<0.01$, and partial $\eta^{2}=0.298$. The quadratic trend was not significant. The pairwise comparisons showed that SP_AVG at the three BAC levels was significantly higher than that at normal level, respectively $(P<0.05)$. There was no significant difference among the three BAC levels.
Mauchly's test of sphericity of SP_SD was not significant, so there is no need of adjustment to the degrees of freedom. Of the within-subjects effects, the main effect of BAC was also statistically significant, $F(3,63)=4.407, P<0.01$, and partial $\eta^{2}=0.173$. As shown in Figure 4(b), SP_SD also increased as a function of BAC, producing a statistically significant linear trend, $F(1,21)=11.383, P<0.01$, and partial $\eta^{2}=0.352$. The quadratic trend was not significant. It was indicated that SP_SD at the BAC levels of $0.06 \%$ and $0.09 \%$ was significantly higher than at the level of $0.00 \%(P<0.05)$. No significance was found for SP_SD among the three BAC levels.

No within-subjects effects were statistically significant for LP_AVG. It was indicated that alcohol did not affect the tendency of vehicle's lateral displacement significantly. Based on the width of road lane, the best value of lane position was $1.875 \mathrm{~m}$. Figure 4(c) showed that drivers when normal driving had a left driving tendency than center of lane, but with right tendency than center of lane when drinking driving.

Mauchly's test of sphericity for LP_SD was statistically significant, and degrees of freedom were also adjusted. The main effect of BAC of the within-subjects effects was statistically significant, $F(1.643,34.498)=4.532, P<0.05$, 


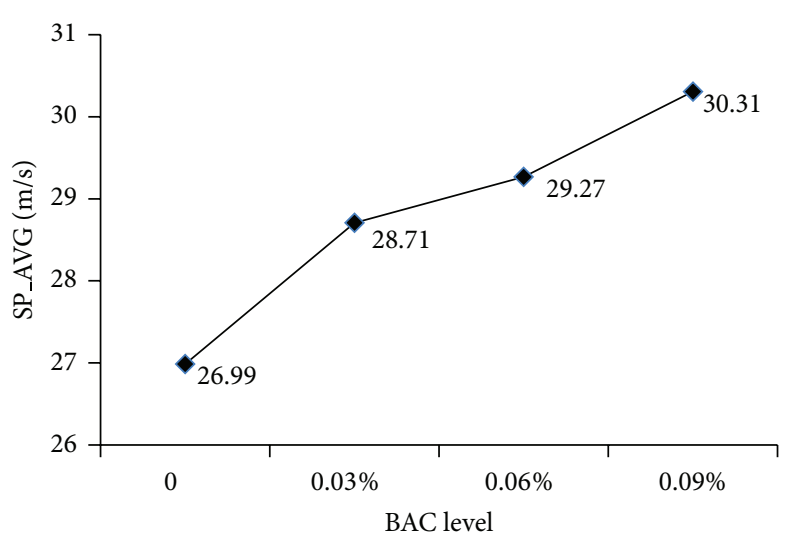

(a) Means of SP_AVG at different BAC levels

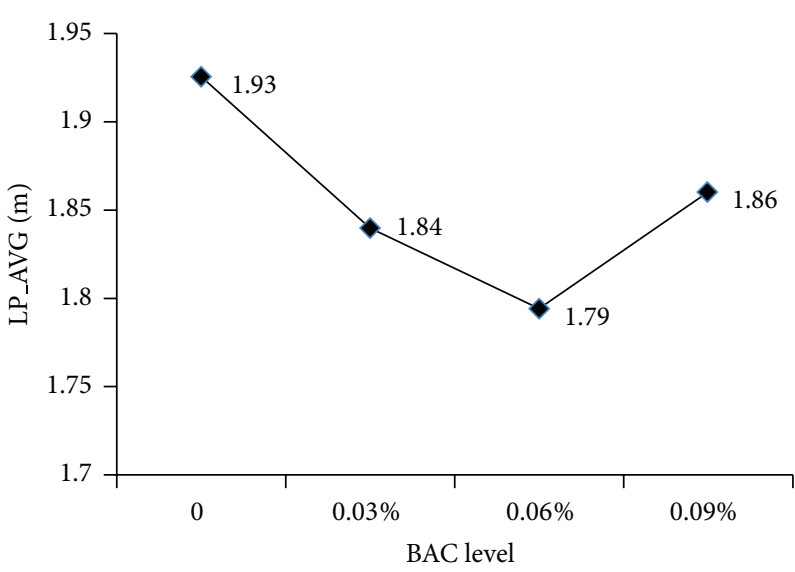

(c) Means of LP_AVG at different BAC levels

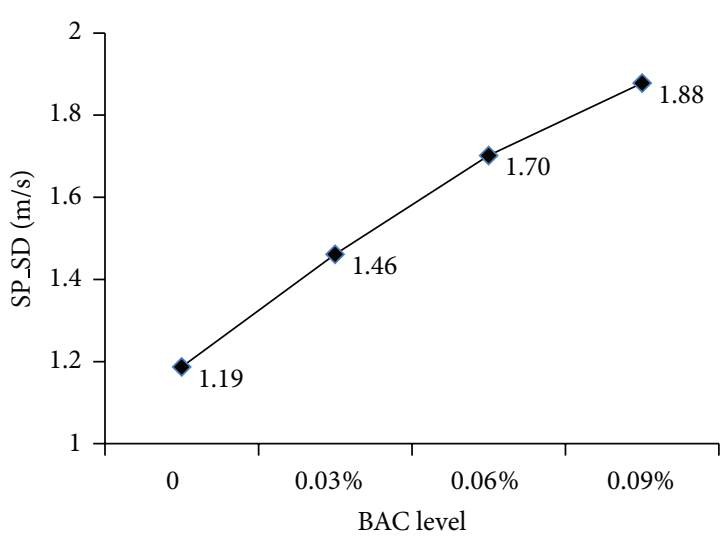

(b) Means of SP_SD at different BAC levels

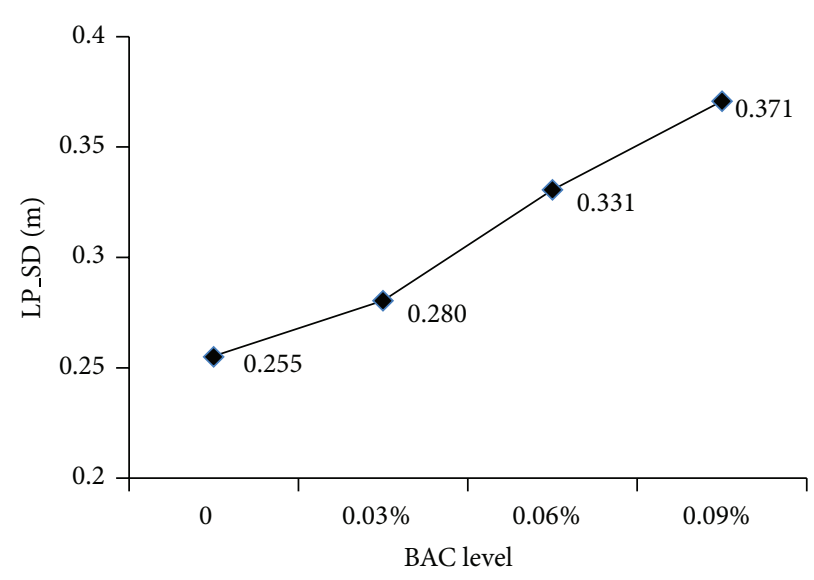

(d) Means of LP_SD at different BAC levels

FIGURE 4: Means of the four indicators at different BAC levels.

and partial $\eta^{2}=0.177$. As shown in Figure 4(d), LP_SD also increased as a function of BAC, producing a statistically significant linear trend, $F(1,21)=6.591, P<0.05$, and partial $\eta^{2}=0.239$. The quadratic trend was not significant. The pairwise comparisons explained that LP_SD at the BAC levels of $0.06 \%$ and $0.09 \%$ was significant higher than it was at the level of $0.00 \%(P<0.05)$. LP_SD at the BAC level of $0.09 \%$ was significantly higher than at the $\mathrm{BAC}$ level of $0.03 \%$. There was no significant difference in other comparisons.

3.4. Discrimination of Driving State. The results of the ANOVA analysis above showed that there are no significant differences of indicators at different BAC levels of drinking driving states. But significant differences were found for some indicators between normal state and drinking driving states. It was indicated that the indicators in urban straight roadway segment might support the discrimination of two states: normal and drinking driving, but they were difficult to support the classification of different BAC levels. To explore the discriminant ability of the driving performance indicators, firstly, the data at the three BAC levels were integrated into one group to distinguish normal state; then, drinking driving states at the three BAC levels were considered one group, respectively, to identify normal state. Therefore, four different discriminant functions were established based on the significant indicators. In the functions, three significant indicators, SP_AVG, SP_SD, and LP_SD, were used to evaluate driving state. Here, $x_{1} \sim x_{3}$ represented the three indicators, $x_{1}$ : SP_AVG, $x_{2}$ : SP_SD, and $x_{3}$ : LP_SD. It was confirmed that each indicator in the drinking driving states and normal states was significantly different. The data met the conditions of using Fisher discriminant method based on the statistical results.

For the discriminant of drinking driving states including all BAC levels and normal states, the standardized discriminant function is

$$
y^{*}=0.341 x_{1}^{*}+0.483 x_{2}^{*}+0.694 x_{3}^{*},
$$

where $y^{*}$ is the discriminant score and $x_{i}^{*}(i=1,2,3)$ means the standardized indicator. The result showed that the discriminant function was statistically significant at the significance level 0.05 .

Discriminant score of each driving state can be calculated through the above function directly. The group centroids of discriminant score for normal states and drinking driving states were -0.610 and 0.212 . A driver's state discriminant score can be calculated by (3), and then drivers' state can be classified based on the score close to which group centroids. 
TABle 3: Accuracy rate of each function.

\begin{tabular}{lcccc}
\hline & Function (1) & Function (2) & Function (3) & Function (4) \\
\hline Accuracy rate of original group & $74.2 \%$ & $63.5 \%$ & $74.3 \%$ & $86.6 \%$ \\
Accuracy rate of cross-validated & $70.6 \%$ & $61.1 \%$ & $72.1 \%$ & $86.6 \%$ \\
\hline
\end{tabular}

Similarly, the standardized discriminant function to identify drinking driving states at BAC level of $0.03 \%$ and normal states is

$$
y^{*}=0.527 x_{1}^{*}+0.27 x_{2}^{*}+0.593 x_{3}^{*} .
$$

The group centroids of discriminant score for normal states and drinking driving states were -0.271 and 0.271 .

The standardized discriminant function to identify drinking driving states at $\mathrm{BAC}$ level of $0.06 \%$ and normal states is

$$
y^{*}=0.131 x_{1}^{*}+0.649 x_{2}^{*}+0.711 x_{3}^{*} .
$$

The group centroids of discriminant score for normal states and drinking driving states were -0.486 and 0.486 .

The standardized discriminant function to identify drinking driving states at BAC level of $0.09 \%$ and normal states is

$$
y^{*}=0.559 x_{1}^{*}+0.269 x_{2}^{*}+0.761 x_{3}^{*} .
$$

The group centroids of discriminant score for normal states and drinking driving states were -0.612 and 0.612 .

Each function was used to classify the corresponding groups of driving states to validate the accuracy. The accuracy rate included classification of original group and crossvalidated, in which each case is classified by the functions derived from all cases other than this case. The accuracy rates of the four functions were shown in Table 3. It showed that the higher the BAC level was, the higher the accuracy rate of the corresponding function was. The accuracy rate of function (3), which was used to classify the normal state group and drinking driving state group with three BAC levels, was close to the middle of the accuracy rates of other three functions.

\section{Discussion}

The effect process of alcohol on drivers is that it firstly affects drivers' physiological characteristics and then affects external performances. It matches the statement that the decline on drivers' bodily functions is the fundamental reason of the impairment of driving skill. The statistical results of the questionnaire showed that most of the participants admitted that they were affected by alcohol on many aspects. Under the influence of alcohol, drivers exhibited the characteristics of being impulsive, sensation-seeking, adventurous, and moving faster. At the same time, the ability of judgment, recognition, reaction, and operation were impaired. Therefore, drinking driving will produce a high probability to serious accidents.

The analysis of accident rate showed that the accident rate increased with the BAC level and it was significantly higher for drinking drivers than for normal drivers. Considering that the accidents happened in urban straight roadway segment without disturbance of other vehicles, the accidents were only related to drivers' driving state. It indicated that even in the simple environment, drinking driving had a high probability to result in an accident. We can deduce that drinking drivers' driving ability will be more difficult to meet the driving needs in a complicated environment and will cause a traffic accident more easily. Although the accident rate may differ from the actual situation, the trend is credible. The result indicated that the accident rate was obviously of positive correlation with drivers' BAC levels.

The analysis of ANOVA with repeated measures proved that SP_AVG, SP_SD, and LP_SD in drinking driving state were all significantly higher than those in normal state. They were related to the changes of drivers' driving attitude and driving ability under the influence of alcohol. Sensationseeking makes drivers show the high-speed traveling state. Because of the decline of perception, more than half of the participants felt the speed slower which was another reason of high-speed travelling. The decline of driving ability decreases the steadiness of vehicle travelling speed. Therefore, the indicator of SP_SD showed significantly higher when drinking driving than normal driving. For lateral movement, although no significant change was found for LP_AVG, LP_SD showed significant differences. The higher LP_SD meant the unsteadiness of lateral movement. In a word, alcohol will cause high vehicle speed and unsteady travelling both in vertical and transverse movement. They are the direct reasons of traffic accident caused by drunk driving. All of the indicators had statistically significant linear trend as the function of BAC. It also proved that driving at higher BAC level would be more dangerous. The significant differences for the indicators of SP_AVG, SP_SD, and LP_SD between drinking driving state and normal state showed that they can contribute to the detection of drinking driving state. Moreover, the significant differences of LP_SD between the BAC levels of $0.03 \%$ and $0.09 \%$ indicated that LP_SD on urban straight roadway segment might support the discrimination of different BAC levels.

Drinking driving will cause significant changes of the indicators of vehicle's travelling condition on urban straight roadway segment. Therefore, we can try to classify the drinking driving state from normal driving state according to the above indicators. The results of Fisher discrimination showed that the function had a certain ability to classify driving state, especially classification of drinking driving states at higher BAC level from normal states. It also indicated that higher BAC level impaired more seriously driving performance and made more differences contrasting with normal driving. At the same time, the imperfection of the classification might be also because that, on one hand, the driving scenario was so simple that the indicators could not fully express enough differences; on the other hand, drivers may have 
obvious individual differences, and it is difficult to set a certain discrimination standards for all drivers. But the result provided reference for discrimination of driving state based on driving performance.

This research revealed drivers' signatures affected by alcohol and tried to classify drinking driving state based on the significant indicators of driving performance. The results have a great potential application in traffic safety research. On one hand, the study on the effects of alcohol is the foundation for the detection of drinking driving. The results revealed the change tendency of drivers' attitude, driving ability, and driving performance under the influence of alcohol, which may be helpful for the countermeasure research of drinking driving. On the other hand, the results can support the study on drivers' driving model, especially for safety driving. Wang et al. [25] have studied the driver's safety approaching behavioral model with various driving characteristics and stated that it could be used in traffic research at the microscopic level. It has also been deeply researched about driver's various information processes and multiruled decision-making mechanism by considering the complicated control process of driving, which was closely related to driving model [26]. Based on these researches, the results about drivers' attitude, driving ability, and driving performance in this study paved the foundation for the study on drivers' drinking driving model, which was very important to improve traffic safety.

There were still some imperfections for the study. First, the measurement of driving performance in this study is not comprehensive. Second, we only analyzed the driving performance data on urban straight roadway segment, which was only a specific road type. However, the driving performance is the basic characteristic of vehicle's travelling and the urban straight roadway segment is the most common road geometry. The study on them is representative and the research method can be generalized. In future studies, the parameter of driving performance should include more aspects, such as brake, accelerator, and steering wheel. The study should also consider more road geometries, such as curve and intersection.

\section{Conclusion}

To explore the impact of alcohol on drivers and driving performance, the experiment was designed to collect 25 participants' subjective feelings and their driving performance data. The distribution of the questionnaire's result was summarized. The accident rate in different BAC levels was statistically analyzed, and ANOVA with repeated measures was used to analyze the signatures of driving performance under the influence of alcohol. Discriminant analysis with significant performance indicators was used to classify drinking driving states from normal driving states. According to the results in this research, the following conclusions can be obtained.

(i) Under the influence of alcohol, most of drivers tend to be more impulsive and adventurous and their abilities of judgment, vigilance, recognition, reaction, and controlling were impaired obviously.

(ii) The accident rate is of positive correlation with BAC level. Driving at higher BAC level will be more dangerous, even in simple driving environment.

(iii) On urban straight roadway segment, SP_AVG, SP_SD, and LP_SD were all significantly higher when drinking driving than those when normal state. They all had statistically significant linear trend as a function of BAC. The three indicators can support the detection of drinking driving state and LP_SD may also contribute to the classification driving states of different BAC levels.

(iv) The above three indicators on urban straight roadway segments can be used to distinguish drinking driving state from normal driving state. The higher the BAC level is, the more accurate the discrimination is.

These conclusions are the basis of the study of drinking driving. They can provide some references for the discrimination of drinking driving state and making countermeasure to it. At the same time, they also support the study of driving safety and the research on drivers' driving model, especially for safety driving.

\section{Conflict of Interests}

The authors declare that there is no conflict of interests regarding the publication of this paper.

\section{Acknowledgments}

This study is supported by the National Natural Science Foundation of China Project, The Study of the Mechanism for Traffic Signs Influence on Driving Behavior and Its Cognitive Model, no. 51108011, and Beijing Natural Science Foundation Project, The Study of Identification Method of Drunk Driving Based on Driving Behavior of Driving Personal Character, no. 8112004.

\section{References}

[1] M. D. Keall, W. J. Frith, and T. L. Patterson, "The influence of alcohol, age and number of passengers on the night-time risk of driver fatal injury in New Zealand," Accident Analysis and Prevention, vol. 36, no. 1, pp. 49-61, 2004.

[2] European Commission, “Saving 20000 lives on our roads," Tech. Rep. COM 2003/311, European Road Safety Action Programme, Luxembourg, 2003.

[3] NHTSA, "Assessing the feasibility of vehicle-based sensors to detect alcohol impairment," Tech. Rep. DOT HS, 811 358, National Highway Traffic Safety Administration, US Department of Transportation, Washington, DC, USA, 2010.

[4] Y. Li, D. Xie, G. Nie, and J. Zhang, "The drink driving situation in China," Traffic Injury Prevention, vol. 13, no. 2, pp. 101-108, 2012.

[5] A. Arnedt, W. Wilde, M. Munt, and M. Maclean, "Simulated driving performance following prolonged wakefulness and 
alcohol consumption: separate and combined contributions to impairment," Journal of Sleep Research, vol. 9, no. 3, pp. 233-241, 2000.

[6] Y.-C. Liu and C. H. Ho, "Effects of different blood alcohol concentrations and post-alcohol impairment on driving behavior and task performance," Traffic Injury Prevention, vol. 11, no. 4, pp. 334-341, 2010.

[7] E. Chamberlain and R. Solomon, "The case for a $0.05 \%$ criminal law blood alcohol concentration limit for driving," Injury Prevention, vol. 8, supplement 3, pp. iiil-iiil7, 2002.

[8] H. Nash, Alcohol and Caffeine: A Study of Their Physiological Effects, Charles C. Thomas, Springfield, Ill, USA, 1962.

[9] NHTSA, "A review of the literature on the effects of low doses of alcohol on driving-related skills," Tech. Rep. HS-809 028, National Highway Traffic Safety Administration, Washington, DC, USA, 2000.

[10] A. J. Adams, B. Brown, G. Haegerstrom-Portnoy, M. C. Flom, and R. T. Jones, "Marijuana, alcohol, and combined drug effects on the time course of glare recovery," Psychopharmacology, vol. 56, no. 1, pp. 81-86, 1978.

[11] NHTSA, "Effects of alcohol on drivers visual information processing," Tech. Rep. PB81-172751, National Highway Traffic Safety Administration, US Department of Transportation, Washington, DC, USA, 1980.

[12] A. M. Williamson, A.-M. Feyer, R. P. Mattick, R. Friswell, and S. Finlay-Brown, "Developing measures of fatigue using an alcohol comparison to validate the effects of fatigue on performance," Accident Analysis and Prevention, vol. 33, no. 3, pp. 313-326, 2001.

[13] M. Linnoila, C. W. Erwin, D. Ramm, and W. P. Cleveland, "Effects of age and alcohol on psychomotor performance of men," Journal of Studies on Alcohol, vol. 41, no. 5, pp. 488-495, 1980.

[14] J. C. Laberg and T. Loberg, "Expectancy and tolerance: a study of acute alcohol intoxication using the balanced placebo design," Journal of Studies on Alcohol, vol. 50, no. 5, pp. 448-455, 1989.

[15] A. M. Smiley, "Marijuana: on-road and driving-simulator studies," Alcohol Drugs Driving, vol. 2, no. 3-4, pp. 121-134, 1986.

[16] E. L. R. Harrison and M. T. Fillmore, "Are bad drivers more impaired by alcohol? Sober driving precision predicts impairment from alcohol in a simulated driving task," Accident Analysis and Prevention, vol. 37, no. 5, pp. 882-889, 2005.

[17] E. L. R. Harrison, C. A. Marczinski, and M. T. Fillmore, "Driver training conditions affect sensitivity to the impairing effects of alcohol on a simulated driving test," Experimental and Clinical Psychopharmacology, vol. 15, no. 6, pp. 588-598, 2007.

[18] M. T. Fillmore, J. S. Blackburn, and E. L. R. Harrison, "Acute disinhibiting effects of alcohol as a factor in risky driving behavior," Drug and Alcohol Dependence, vol. 95, no. 1-2, pp. 97106, 2008.

[19] C. T. Nagoshi, J. R. Wilson, and L. A. Rodriguez, "Impulsivity, sensation seeking, and behavioral and emotional responses to alcohol," Alcoholism, vol. 15, no. 4, pp. 661-667, 1991.

[20] D. R. Mayhew, A. C. Donelson, D. J. Beirness, and H. M. Simpson, "Youth, alcohol and relative risk of crash involvement," Accident Analysis and Prevention, vol. 18, no. 4, pp. 273-287, 1986.

[21] P. L. Zador, "Alcohol-related relative risk of fatal driver injuries in relation to driver age and sex," Journal of Studies on Alcohol, vol. 52, no. 4, pp. 302-310, 1991.
[22] F. Bella, "Validation of a driving simulator for work zone design," Transportation Research Record, no. 1937, pp. 136-144, 2005.

[23] F. Bella, "Driving simulator for speed research on two-lane rural roads," Accident Analysis and Prevention, vol. 40, no. 3, pp. 10781087, 2008.

[24] P. E. Watson, "Total body water and blood alcohol levels: updating the fundamentals," Human Metabolism of Alcohol, vol. 1, pp. 41-58, 1989.

[25] W. Wang, W. Zhang, H. Guo, H. Bubb, and K. Ikeuchi, "A safetybased approaching behavioural model with various driving characteristics," Transportation Research C, vol. 19, no. 6, pp. 1202-1214, 2011.

[26] W. Wang, Y. Mao, J. Jin et al., "Driver's various information process and multi-ruled decision-making mechanism: a fundamental of intelligent driving shaping model," International Journal of Computational Intelligence Systems, vol. 4, no. 3, pp. 297-305, 2011. 


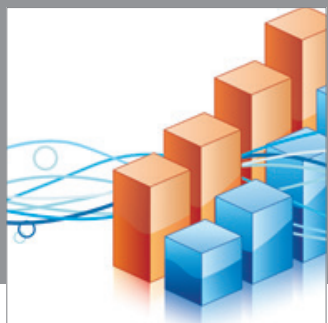

Advances in

Operations Research

mansans

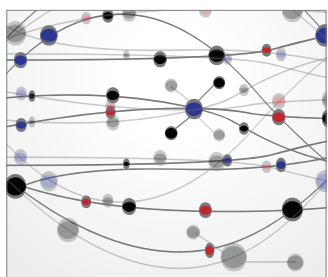

The Scientific World Journal
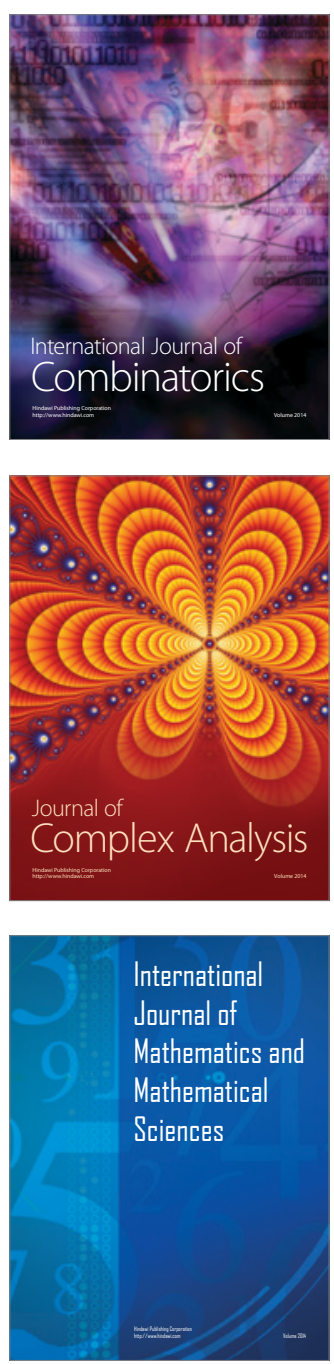
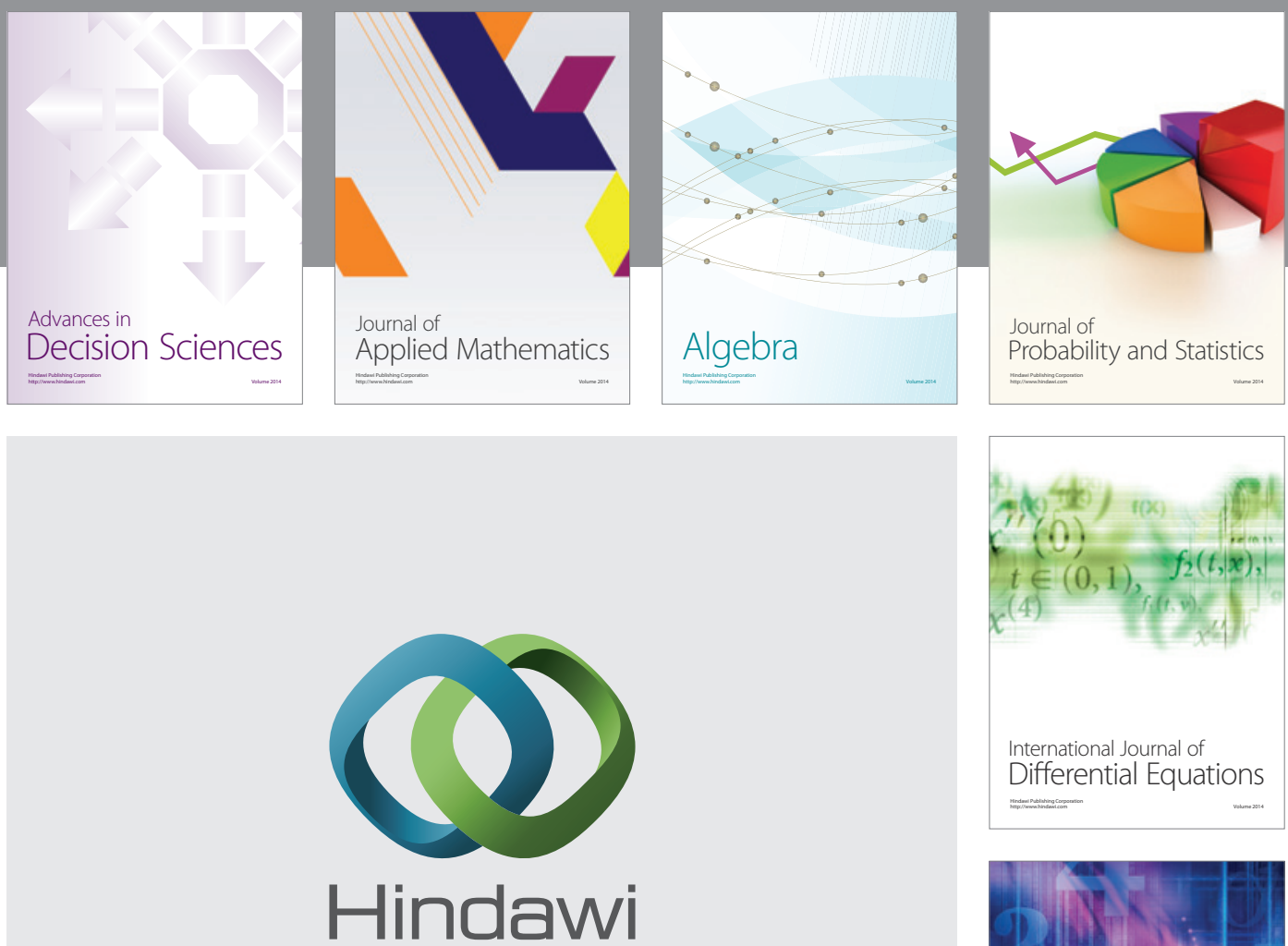

Submit your manuscripts at http://www.hindawi.com
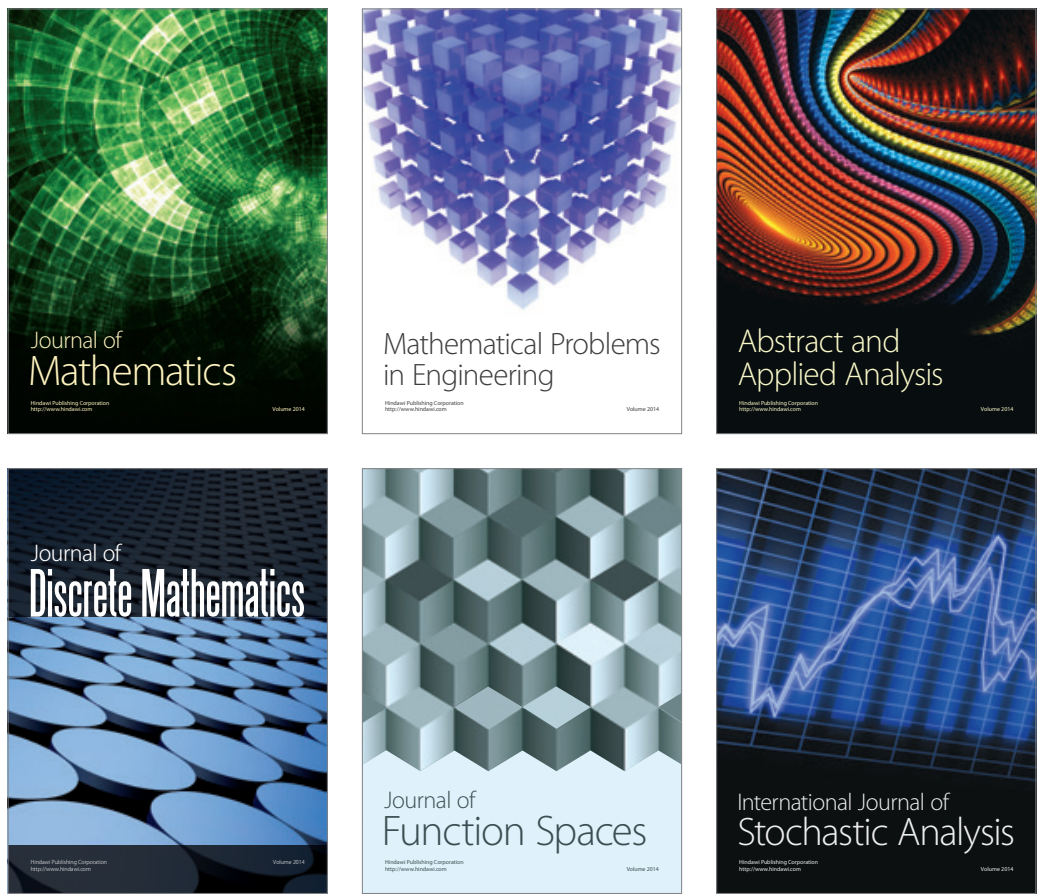

Journal of

Function Spaces

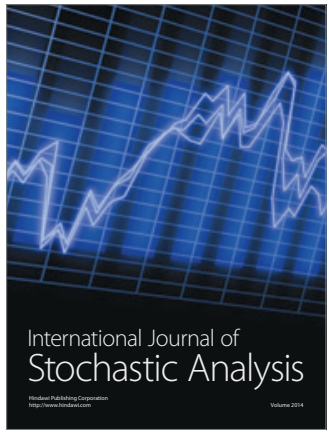

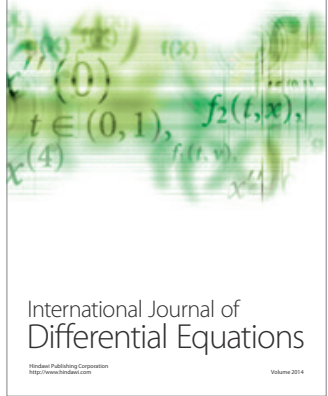
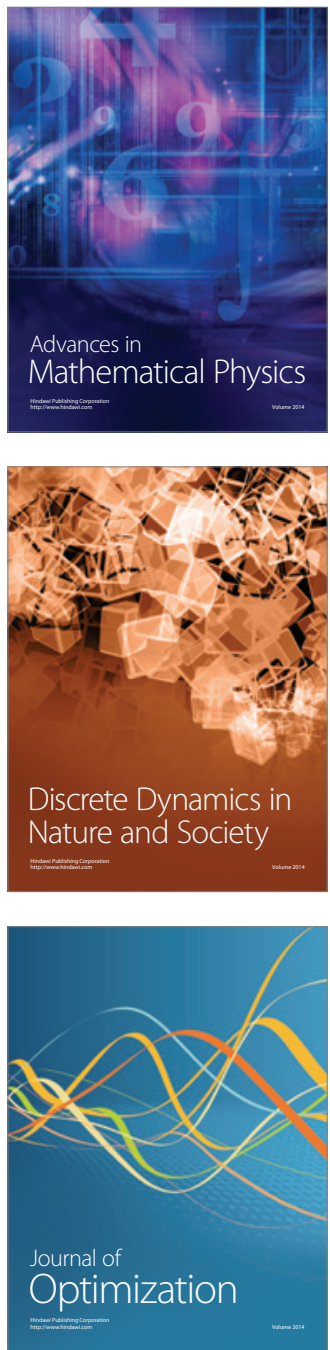